\title{
Correction to: Central Blockade of E-Prostanoid 3 Receptor Ameliorated Hypertension Partially by Attenuating Oxidative Stress and Inflammation in the Hypothalamic Paraventricular Nucleus of Spontaneously Hypertensive Rats
}

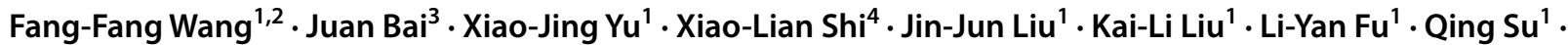

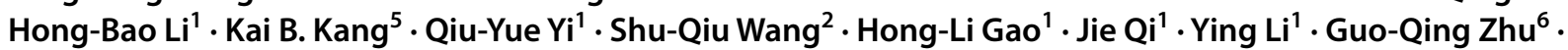 \\ Yu-Ming Kang ${ }^{1}$
}

Published online: 5 February 2021

(c) Springer Science+Business Media, LLC, part of Springer Nature 2021

\section{Correction to: Cardiovascular Toxicology https://doi.org/10.1007/s12012-020-09619-w}

The original version of this article unfortunately contained a mistake in the name of one co-author. The correct name of the co-author "Juan Ba" should be "Juan Bai".
Publisher's Note Springer Nature remains neutral with regard to jurisdictional claims in published maps and institutional affiliations.
The original article can be found online at https://doi.org/10.1007/ s12012-020-09619-w.

Ying Li

lyinxian777@mail.xjtu.edu.cn

$\triangle \quad$ Yu-Ming Kang

ykang@mail.xjtu.edu.cn

1 Department of Physiology and Pathophysiology, Xi'an Jiaotong University School of Basic Medical Sciences, Shaanxi Engineering and Research Center of Vaccine; Key Laboratory of Environment and Genes Related to Diseases of Education Ministry of China, Xi' an Jiaotong University, Xi' an 710061, China

2 Department of Functional Medicine, School of Basic Medical Sciences, Jiamusi University, Jiamusi 154007, China
3 Department of Anesthesiology, Center for Brian Science, The First Affiliated Hospital of Xi' an Jiaotong University, Xi' an 710061, China

4 Department of Pharmacology, School of Basic Medical Sciences, Xi'an Jiaotong University Health Science Center, Xi' an 710061, China

5 Department of Ophthalmology and Visual Sciences, University of Illinois at Chicago, Chicago, IL, USA

6 Key Laboratory of Cardiovascular Disease and Molecular Intervention, Department of Physiology, Nanjing Medical University, Nanjing 210029, China 